\title{
Effect of ACEI and ARB treatment on nitric oxide-dependent endothelial function
}

\author{
Kangbo Li $i^{1,2}$, Claudia Zemmrich ${ }^{3}$, Peter Bramlage ${ }^{3}$, Anja Bondke Persson ${ }^{2}$, Mesud Sacirovic ${ }^{1}$, \\ Oliver Ritter ${ }^{4,5}$, Eva Buschmann ${ }^{6}$, Ivo Buschmann ${ }^{1,5}$, and Philipp Hillmeister ${ }^{1,5}$

\footnotetext{
Department for Angiology, Center for Internal Medicine I, Brandenburg Medical School Theodor Fontane, Campus University Clinic Brandenburg, Deutsches Angiologie Zentrum Brandenburg-Berlin (DAZB), Brandenburg an der Havel, Germany

Campus University Clinic Brandenburg, Brandenburg an der Havel, Germany School Theodor Fontane and the University of Potsdam, Brandenburg Medical School Theodor Fontane, Germany
} \\ ${ }^{2}$ Charité - Universitätsmedizin Berlin, corporate member of Freie Universität Berlin and Humboldt-Universität zu Berlin, Germany \\ ${ }^{3}$ Institute for Pharmacology and Preventive Medicine, Cloppenburg, Germany \\ ${ }^{4}$ Department for Cardiology, Center for Internal Medicine I, Brandenburg Medical School Theodor Fontane, \\ ${ }^{5}$ Faculty of Health Sciences, joint Faculty of the Brandenburg University of Technology Cottbus - Senftenberg, the Brandenburg Medical \\ ${ }^{6}$ Department of Cardiology, University Clinic Graz, Austria
}

\begin{abstract}
Summary: Background: Angiotensin-converting-enzyme inhibitors (ACEI) and angiotensin II receptor blockers (ARB) are widely used as a first-line therapy for the treatment of cardiovascular disease. Here, ACEI modulate the bradykinin receptor (BDKRB1 and BDKRB2) system and NO-dependent endothelial function, thus determining cardiovascular health and regenerative arteriogenesis. The current study aims at evaluating nitric oxide-dependent endothelial function, and gene expression of bradykinin receptors in peripheral blood mononuclear cells (PBMC) from patients with ACEI or ARB treatment. Patients and methods: The WalkByLab has been established to screen cardiovascular patients for peripheral artery disease and coronary artery disease. In total 177 patients from WalkByLab with heterogenous disease and risk status were randomly selected, divided according to their medication history into the following groups: 1. ACEl group, 2. ARB group or 3. non-ACE/ARB group. Total plasma nitrite/nitrate (NO) levels were measured, endothelial function was evaluated by assessing flow meditated dilation (FMD). PBMC were isolated from peripheral whole blood, and gene expression (qRT-PCR) of bradykinin receptors and angiotensin converting enzyme were assessed. Results: Plasma total NO concentration in the ACEI group (24.66 $\pm 16.28, \mu \mathrm{mol} / \mathrm{l})$ was increased as compared to the ARB group $(18.57 \pm 11.58, \mu \mathrm{mol} / \mathrm{l}, P=0.0046)$ and non-ACE/ARB group $(16.83 \pm 8.64, \mu \mathrm{mol} / \mathrm{l}, P=0.0127)$ in patients between 40 to 90 years of age. However, FMD values (\%) in the ACEl group $(7.07 \pm 2.40$, \%) were similar as compared to the ARB $(6.35 \pm 2.13, \%)$ and non-ACE/ARB group $(6.51 \pm 2.15, \%)$, but significantly negatively correlated with age. Interestingly, BDKRB1 mRNA level was significantly higher and BDKRB2 mRNA level lower in the ACEI group (BDKRB1 3.88-fold \pm 1.05 , BDKRB2 0.22-fold \pm 0.04 ) as compared to the non-ACE/ARB group (BDKRB1 1.00-fold $\pm 0.39, P<0.0001, B D K R B 21.00$-fold $\pm 0.45, P=0.0136$ ). Conclusions: ACEl treatment enhances total nitrite/nitrate concentration, furthermore, upregulates BDKRB1 in PBMC, but downregulates BDKRB2 mRNA expression. FMD is a strong determinant of vascular aging and is sensitive to underlying heterogenous cardiovascular diseases.
\end{abstract}

Keywords: flow mediated dilation, nitric oxide, kallikrein-kinin-system, angiotensin-converting-enzyme inhibitors, angiotensin receptor blockers

\section{Introduction}

Vascular aging processes impact atherosclerosis risk and arteriogenic remodelling capacity and are most commonly analyzed by measuring peripheral blood mononuclear cells (PBMCs) phenotype and endothelial function [1]. Whereas atherosclerosis and arterial occlusion are the consequence of most vascular aging processes, regenerative arteriogenesis is the primary compensatory mechanism against ischemic vascular disease (CAD, PAD). Arteriogenesis is one of the key mechanisms of protective vascular growth, and involves the remodeling and positive outgrowth of 
pre-existing collateral arteries following a stenosis or arterial occlusion [2]. For arteriogenesis it was shown as well that endothelial function and PBMCs play a critical role in endothelial proliferation, intima formation and maturation of collateral arteries $[3,4]$. Thus, where vascular regeneration interacts positively with PBMC phenotypes and endothelial function, these factors are negatively influenced by pathological processes of aging, and determine the severity of diseases. Recent research has shown that in terms of vascular regeneration, PBMC activity and endothelial function are linked through bradykinin receptor signaling and NO production [1, 5]. In regard to vascular diseases, Angiotensin-converting-enzyme inhibitors (ACEI) and angiotensin II receptor blockers (ARB) are cornerstones of the pharmacological therapy for patients with coronary artery disease $(\mathrm{CAD})$, peripheral artery disease $(\mathrm{PAD})$, heart failure (HF) and essential hypertension $[6,7,8,9]$. Furthermore, ACEI and ARB are discussed to be involved in arteriogensis and vascular regeneration. Therefore, in this work, we investigate whether ACEI and ARB therapy is associated with bradykinin receptor signaling in PBMCs, NO production, and endothelial function.

Beyond the property that angiotensin-converting enzyme (ACE) inhibitors are used to treat hypertension and ischemic heart disease, many research groups are looking at the bradykinin-mediated aspects of ACE inhibition [10]. Angiotensin-converting enzyme (ACE, also known as kininase II) is the key enzyme for the degradation of bradykinin (BK). BK is one of the major endotheliumdependent vasodilators in cardiovascular homeostasis. Therefore ACEI, the inhibitor of ACE, prevents BK degradation and leads to the BK mediated and endotheliumdependent vasodilation (EDV) [11]. Furthermore, our group was the first to demonstrate that bradykinin receptor signaling is a strong modulator of therapeutic arteriogenesis [4]. Research has shown that ACEI can be regarded as the allosteric enhancer of bradykinin receptors. The bradykinin receptor family consists of two $G$ protein-coupled receptors: bradykinin receptor 1 (BDKRB1) and bradykinin receptor 2 (BDKRB2), whereby ACEI are agonists activating BDKRB1 directly on the extracellular loop and also indirectly activating the $\mathrm{ACE} / \mathrm{BDKRB} 2$ heterodimer by altering conformational change [12]. Bradykinin receptor BDKRB2 is constitutively expressed in various cell types, whereas bradykinin receptor BDKRB1 is only upregulated under the inflammatory conditions. BK and kallidin, derived from plasma and tissue kallikrein, respectively, are the corresponding ligands of BDKRB2, whereas the BK and kallidin derivatives des-Arg9-bradykinin and Lys-bradykinin are the corresponding ligands of BDKRB1 [13]. It was shown that BDKRB2 and BDKRB1 signaling induces nitric oxide (NO) production in endothelial cells and circulating immune cells [14]. Hence, bradykinin receptors and NO are major players in mediating arteriogenesis and endothelial function [5].

In addition to ACEI, ARB are recommended as first-line treatment options for hypertension. ARB are superior to
ACEI in regard to their more favorable (drug) safety profile ARB selectively inhibit angiotensin II type 1 receptors (AT1), thereby augmenting angiotensin II type 2 receptor (AT2) activity, whose effects antagonize AT1 [15, 16]. Interestingly, a functional heterodimerization of AT2-BDKRB2 enhances peptide ligand binding and receptor activation [17]. Animal experiments also demonstrated that angiotensin II induced upregulation of BDKRB1 is abrogated in AT1 knockout mice. BDKRB1 can be regarded as the downstream target of AT1 agonists [18].

In the current study we therefore analyze total nitrite/ nitrate concentration in plasma, endothelial function by flow mediated dilation and gene expression of bradykinin receptors and angiotensin converting enzyme in PBMCs from patients with $\mathrm{CAD}, \mathrm{PAD}$, cerebrovascular disease (CVD), and hypertension treated pharmacologically with either ACEI or ARB.

\section{Patients and methods}

\section{Study population}

In total 177 patients with atherosclerotic disease (CAD, PAD, CVD) were randomly selected from the WalkByLab-Registry database. These are the patients who presented to the WalkByLab center Brandenburg/Havel (Brandenburg Clinic, Brandenburg Medical School) Germany in the period from July 2019 to February 2020 with the corresponding pre-existing cardiovascular disease. The WalkyByLab aims to interdisciplinarily screen, diagnose and follow-up patients with cardiovascular disease (www.lauflab.de). The WalkByLab register trial protocol was reviewed and approved by the ethical committee of the Cottbus Medical Association (Landesärztekammer Cottbus, study number of the ethics committee: AS $74(\mathrm{bB}) / 2018)$. The screening trial is performed in accordance with the principles of the declaration of Helsinki.

\section{Isolation of peripheral blood mononuclear cells}

$15 \mathrm{ml}$ blood was collected in three vacutainer EDTA tubes (Becton Dickinson) by routine venipuncture procedure. Blood was diluted 1:1 in PBS, and carefully layered onto the Ficoll-Paque density gradient media (GE Healthcare) at a ratio of $4: 3$ and centrifuged at $400 \mathrm{~g}$ for $25 \mathrm{~min}$. The upper layer of plasma was collected from each sample and frozen in $2 \mathrm{ml}$ aliquots at $-80^{\circ} \mathrm{C}$ until use for determination of total nitrite/nitrate levels.

The middle layer containing PBMCs was transferred to $50 \mathrm{ml}$ sterile tubes, and washed twice with $40 \mathrm{ml}$ PBS. Cell counting was performed under the microscope. Aliquots including 5 million cells were centrifuged at $1500 \mathrm{~g}$ for 10 minutes and the pellet was frozen and stored at $-80{ }^{\circ} \mathrm{C}$ until use for RNA isolation. 


\section{RNA isolation and quantitative real-time PCR-based analysis}

Total RNA was extracted from PBMCs by using the Trizol reagent (Thermo Fisher Scientific) according to the manufacturer's instructions. RNA integrity was assessed by agarose gel electrophoresis, quantitative analysis of RNA was performed using Qubit ${ }^{\circledR}$ RNA BR assay together with the Qubit $^{\circledR}$ 3.0 Fluorometer (Thermo Fisher Scientific). $1 \mu \mathrm{g}$ total RNA was used for the first strand cDNA synthesis by using QuantiTect Reverse Transcription Kit (QIAGEN). Samples were deployed in the peqSTAR thermal cycler (VWR International). The mRNA levels of the target gene were analyzed by real-time polymerase chain reaction using the LightCycler ${ }^{\circledR} 96$ Real-Time PCR System (Roche). Each reaction system contained $1 \mu \mathrm{l}$ cDNA, $2 \mu \mathrm{l}$ primer working solution, $7 \mu \mathrm{l}$ RNase/DNase-free water and $10 \mu \mathrm{l}$ QuantiTect $^{\circledR}$ SYBR $^{\circledR}$ Green PCR Kit (QIAGEN). All the primers were synthesized and purchased from Eurofins Genomics Germany GmbH. All samples were run in duplicate (Table I).

\section{Determinations of total nitrite/nitrate levels in plasma}

In the cell, nitric oxide is converted into nitrite and nitrate as the stable metabolites after a series of reactions. Therefore, total nitrite/nitrate concentration is the best value for assessing the NO production in a physiological system. Plasma samples were thawed and ultrafiltered to remove hemoglobin by centrifugation in an ultra- 0.5 centrifugal filter devices (Merck Millipore). Total nitrite/nitrate levels in plasma were determined according to the manufacturer's instructions (Cayman Chemical). In brief, conversion of nitrate to nitrite is achieved by utilizing nitrate reductase enzyme. During this process a deep purple azo dye is formed based on Griess reagent, and the light absorbance can be spectrophotometrically measured at $548 \mathrm{~nm}$ on a Spark multimode microplate reader (Tecan Group AG).

\section{Assessment of flow mediated dilation}

The AngioDefender (Everist Health) medical device was used to evaluate endothelial function by assessing flow mediated dilation (FMD) of the brachial artery. FMD measurement is regarded as the gold standard method for evaluation of endothelial function. The novel Angio Defender medical device allows for a precise, standardized, and automated measurement of FMD-value by oscillation technique, it has been proven to show less measurement errors when compared with classical FMD measurement based on ultrasound techniques. The AngioDefender measurement technique is therefore regarded superior to the classical measuring methods.

\section{Treadmill testing}

A treadmill and the "Gardner Test Protocol" are used to determine the maximum walking distance. The test is performed under the supervision of a physician, the patient is secured with a drop stop device (safety bar with chest harness). The patient initially stands on the lateral treads and the natural walking speed is tested, while the treadmill accelerates to $3.2 \mathrm{~km} / \mathrm{h}$ to allow the patient to enter the treadmill and start the test (time "zero"). Subsequently, the incline angle of the treadmill changes by 2 degrees every 2 minutes. In addition to this, the concomitant symptoms (peripheral fatigue, angina pectoris, dyspnea and others) during treadmill testing have also been recorded. However, some patients did not participate to the walking test because they suffered severe cardiovascular disease. Hence, we have results from in total 83 patients of the here analyzed patient population.

\section{Statistical analysis}

Data of demographics, plasma nitrite level and flow mediated dilation are given as mean \pm standard deviation (SD), data of mRNA expression are given as mean \pm standard error of the mean (SEM). A Kolmogorov-Smirnov test was carried out to check the distribution of quantitative variables, data following a normal distribution were analyzed by the one-way analysis of variance (ANOVA), data following a non-normal distribution were analyzed by the Kruskal-Wallis test. A p-value of $P \leq 0.05$ (two-sided) was considered to indicate statistical significance.

\section{Results}

All patients were screened for medication history, blood samples were taken, and FMD measured. Patients were divided into three groups as follows according to the medication history: 1. ACEI group, 2. ARB group, and 3. non-ACE/ARB group (neither treated with ACEI or ARB). Population characteristics are presented in Table II.

Patient age groups were 40 to 49 years (2.82\%), 50 to 59 years $(9.60 \%), 60$ to 69 years $(36.72 \%), 70$ to 79 years (31.64\%) and 80 to 89 years (19.21\%), respectively. The mean age was 70.10 years $(\mathrm{SD}=9.35) .41 .81 \%$ patients were female and $58.19 \%$ patients were male. The mean BMI of patients was $27.96(\mathrm{SD}=4.95)$ and of these patients were normal weight $(\mathrm{BMI}=18.5-23,27.81 \%)$, overweight $(\mathrm{BMI}=$ 23.0-27.49, 44.38\%), and obese (BMI>27.5, 27.81\%), respectively. Common comorbidities included: hypertension $(n=167)$, diabetes mellitus $(n=36)$, renal dysfunction $(n=34)$, hyperlipidemia $(n=74) .84$ patients suffered from PAD, 91 patients suffered from CAD, and 12 patients suffered from CVD. For 32 patients a history of myocardial infarction was reported, heart failure for 41 patients, 74 were smokers.

\section{Total plasma nitrite/nitrate concentration}

Total plasma nitrite/nitrate level in the ACEI group $(24.66 \pm 16.28, \mu \mathrm{mol} / \mathrm{l})$ was significantly higher than that 
Table I. qRT-PCR primer list (sense and antisense primers)

\begin{tabular}{lll}
\hline Gene & Forward (5'-3') & Reverse (5'-3') \\
\hline BDKRB1 & ATTCTCCCACCTCAGCCTCT & CTCTGGTTGGAGGATTGGAG \\
BDKRB2 & CTTCATGGCCTACAGCAACA & GCACACTCCCTGGTACACCT \\
ACE & ATGAAGACCTGTTATGGGCATGG & ATTTCGGGTAAAACTGGAGGATGG \\
RPLPO & ACGGGTACAAACGAGTCCTG & AGCCACAAAGGCAGATGGAT \\
\hline
\end{tabular}

Table II. Population characteristics

\begin{tabular}{|c|c|c|c|c|}
\hline & ACEI $(n=73)$ & ARB $(n=68)$ & Non-ACEI/ARB $(n=36)$ & $P$ \\
\hline \multicolumn{5}{|l|}{ Demographics } \\
\hline Gender, male & 47 & 34 & 22 & 0.207 \\
\hline Age, y & $68.45 \pm 9.40$ & $71.85 \pm 9.09$ & $70.11 \pm 9.38$ & 0.097 \\
\hline $\mathrm{BMI}, \mathrm{kg} / \mathrm{m}^{2}$ & $27.94 \pm 5.34$ & $28.62 \pm 5.11$ & $26.69 \pm 3.45$ & 0.181 \\
\hline \multicolumn{5}{|l|}{ Medical history } \\
\hline Hypertension & 67 & 67 & 33 & 0.164 \\
\hline Diabetes mellitus & 16 & 12 & 8 & 0.781 \\
\hline Renal dysfunction & 12 & 13 & 9 & 0.566 \\
\hline Hyperlipidemia & 32 & 30 & 12 & 0.513 \\
\hline PAD & 38 & 30 & 16 & 0.590 \\
\hline CAD & 38 & 33 & 20 & 0.784 \\
\hline CVD & 6 & 4 & 2 & 0.814 \\
\hline Myocardial infarction & 8 & 16 & 8 & 0.118 \\
\hline Heart failure & 18 & 16 & 7 & 0.828 \\
\hline $\mathrm{PCl} / \mathrm{PPI}$ & 27 & 28 & 18 & 0.431 \\
\hline CABG/PABG & 8 & 10 & 1 & 0.174 \\
\hline Smoking & 28 & 28 & 18 & 0.506 \\
\hline \multicolumn{5}{|l|}{ Medication history } \\
\hline Beta-blocker & 42 & 38 & 23 & 0.725 \\
\hline Calcium channel blockers & 22 & 33 & 7 & 0.007 \\
\hline Diuretics & 31 & 24 & 6 & 0.028 \\
\hline Statins & 46 & 45 & 23 & 0.924 \\
\hline Aspirin & 36 & 42 & 16 & 0.169 \\
\hline Antidiabetics & 10 & 9 & 4 & 0.928 \\
\hline Insulin & 6 & 5 & 3 & 0.977 \\
\hline
\end{tabular}

PCl: Percutaneous coronary intervention, PPI: percutaneous peripheral interventional, CABG: coronary artery bypass grafting, PABG: peripheral artery bypass grafting.

in the ARB group $(18.57 \pm 11.58, \mu \mathrm{mol} / \mathrm{l}, P=0.0046)$ and non-ACE/ARB group $(16.83 \pm 8.64 \mu \mathrm{mol} / \mathrm{l}, \quad P=0.0127)$ (Figure 1A). The plasma total nitrite/nitrate level in the ARB group showed a similar concentration $(18.57 \pm 1$ $1.58, \mu \mathrm{mol} / \mathrm{l})$ as compared to the non-ACE/ARB group $(16.83 \pm 8.64 \mu \mathrm{mol} / \mathrm{l})$.

Furthermore, plasma nitrite/nitrate levels were analyzed in regard to possible sex differences in the medical treatment groups, however results showed no relation between plasma nitrite/nitrate and sex $(P=0.963)$ (Figure 1B). A Spearman's rank correlation was calculated in order to investigate how plasma total nitrite/nitrate is related to the different medical treatment groups in the context with age. Nitrite/nitrate was not correlated with age in any group $(P=0.215)$ (Figure 1C).

\section{Endothelial function analyzed by flow mediated dilation (FMD)}

Cardiovascular health and arteriogenic capacity are largely determined by arterial endothelial function. Endothelial function was assessed in all patients using the AngioDefender, which is a new and standardized method for determining FMD. FMD in the ACEI group $(7.07 \pm 2.40, \%)$ showed values with no significant differences as compared to the ARB group $(6.35 \pm 2.13, \%)$ and values were comparable to the non-ACEI/ARB group (6.51 \pm 2.15 , \%) (Figure 2A). FMD was analyzed in regard to possible sex differences and results showed no relation between FMD and gender $(P=0.229)$ (Figure 2B). Correlation analysis of FMD with age showed a significant and negative correlation. FMD 
A

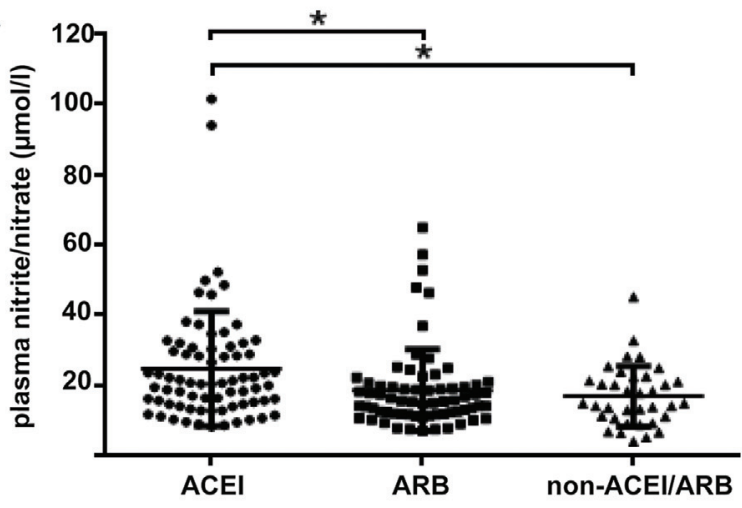

B

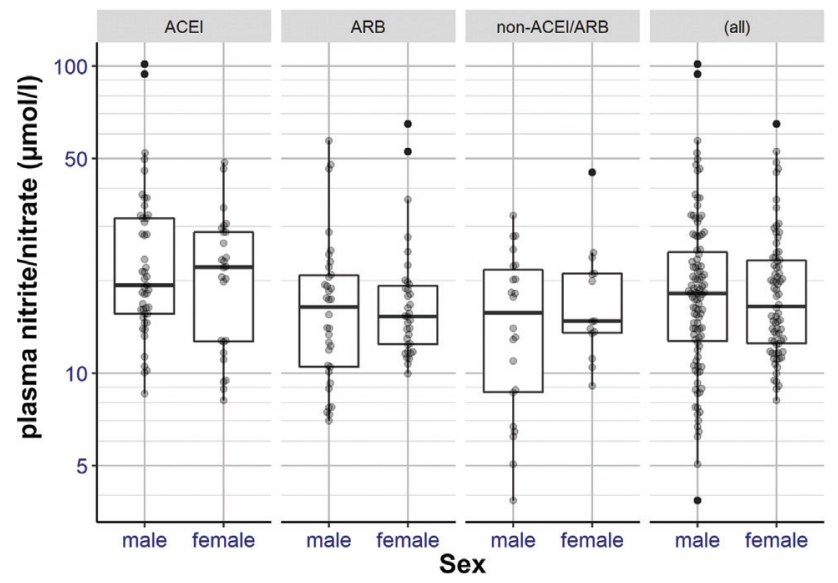

C

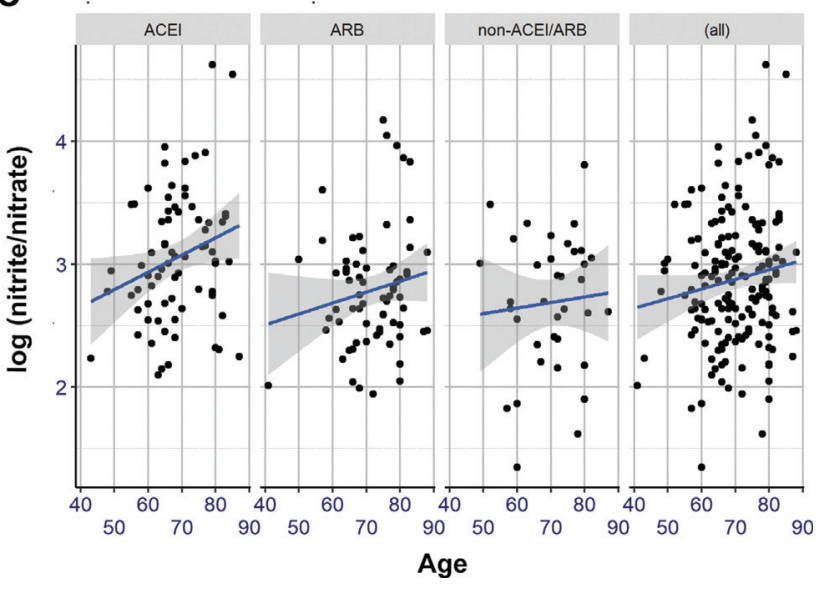

Figure 1. (A) Plasma total nitrite/nitrate levels in patients of three groups $\left({ }^{*} P \leq 0.05\right)$. (B) Correlation analysis between plasma total nitrite/nitrate levels and gender shows no correlation. (C) Correlation analysis between plasma total nitrite/nitrate levels (log scale) and age shows no correlation. ACEI $(n=73)$, ARB $(n=68)$, non-ACEI/ARB $(n=36)$.

decreases with increasing age in all medical treatment groups $(P<0.001)$ (Figure $2 \mathrm{C}$ ). Furthermore, FMD does not correlate with nitrite/nitrate level in any medical treatment group $(P=0.949)$ (Figure 3A, Table III)

\section{Treadmill testing}

Treadmill testing results showed that the maximal walking distance and walking speed in the ACEI are slightly higher than in the ARB group and slight lower than in the nonACEI/ARB but without significant differences. The results of concomitant symptoms reported during treadmill testing

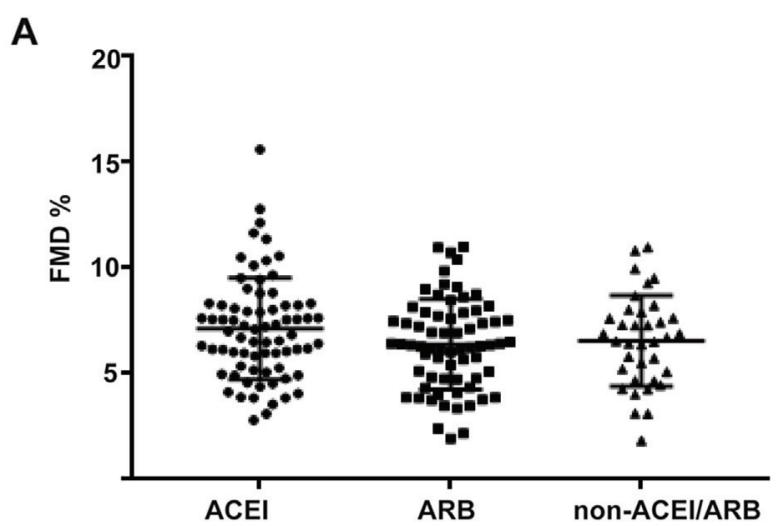

B
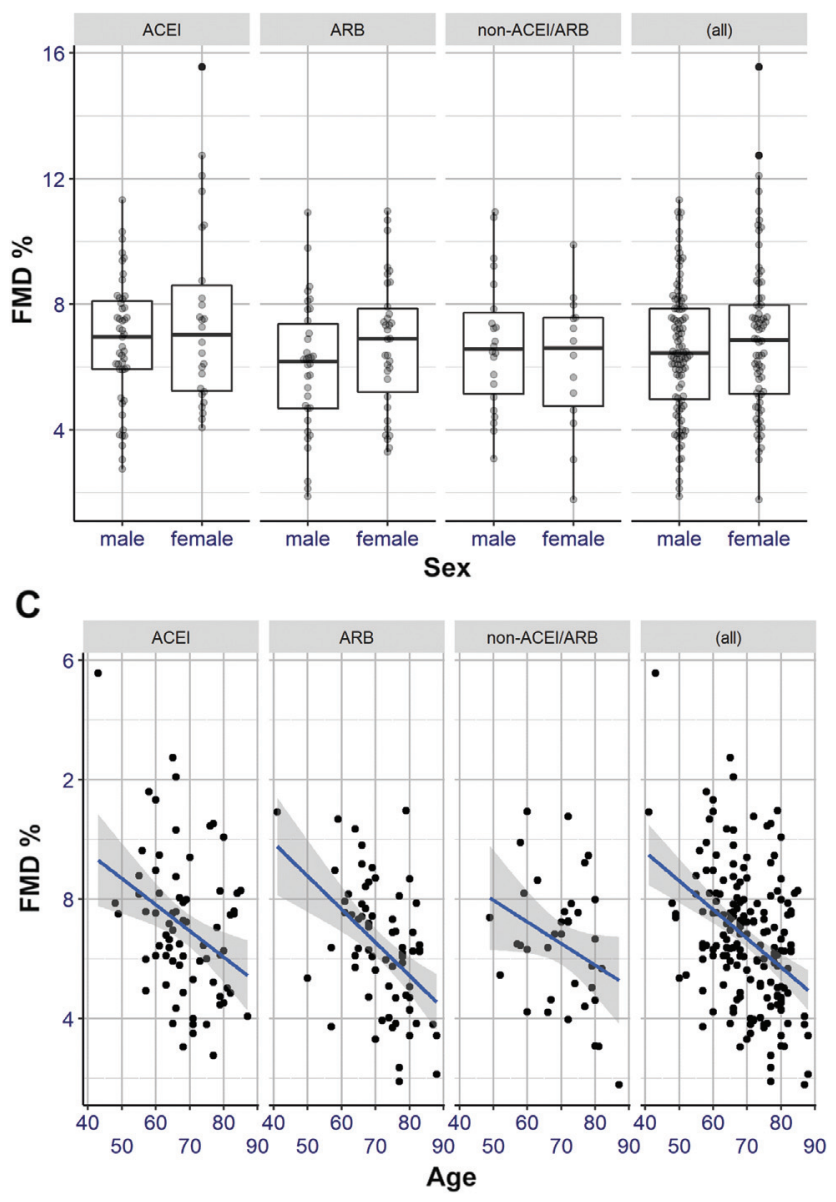

Figure 2. (A) FMD of the brachial artery in patients of three groups. (B) Correlations analysis between FMD and gender shows no correlation. (C) FMD correlates with age in all treatment groups (linear model). ACEI $(n=73)$, ARB $(n=68)$, non-ACEI/ARB $(n=36)$.

showed there is no significant differences between three groups groups (Table IV).

\section{Gene expression analysis of markers and regulators of arteriogenesis}

ACEI treatment resulted in an upregulation of BDKRB1 in PBMCs, but a downregulation of BDKRB2 mRNA expression. BDKRB1 mRNA level was significantly higher in the ACEI group (3.88-fold \pm 1.05 ), when compared to nonACE/ARB group (1.00-fold $\pm 0.39, P<0.0001)$. The expression level of BDKRB1 in the ARB group (2.46-fold \pm 0.59 ) 
A

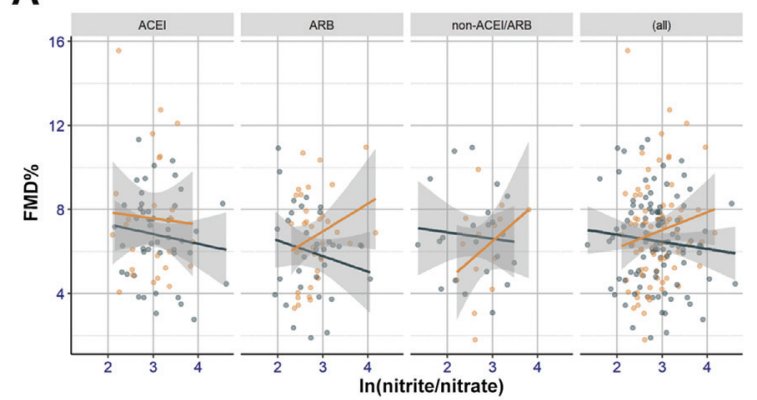

B

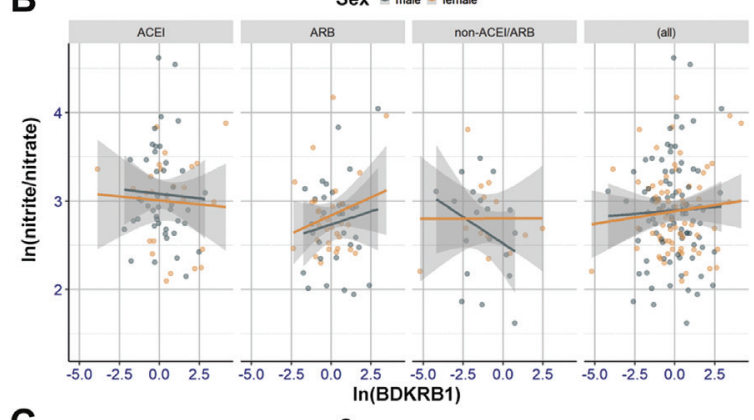

C

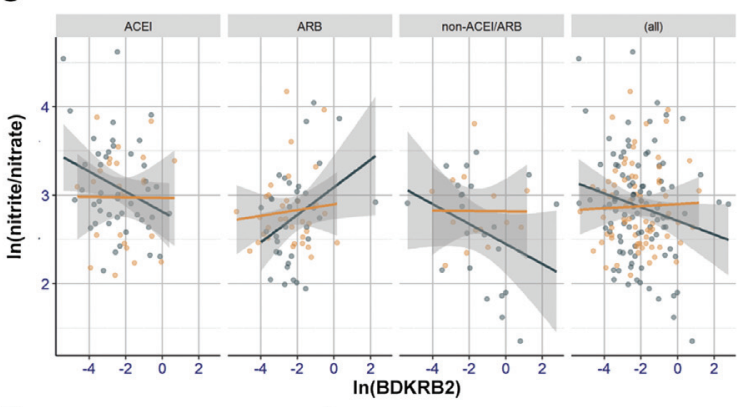

D

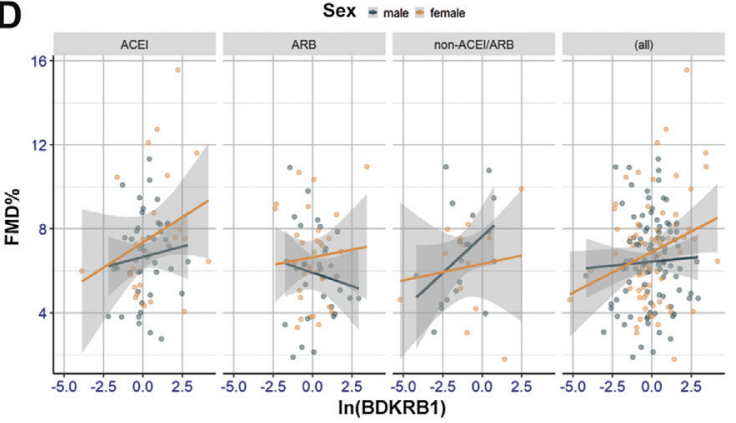

E

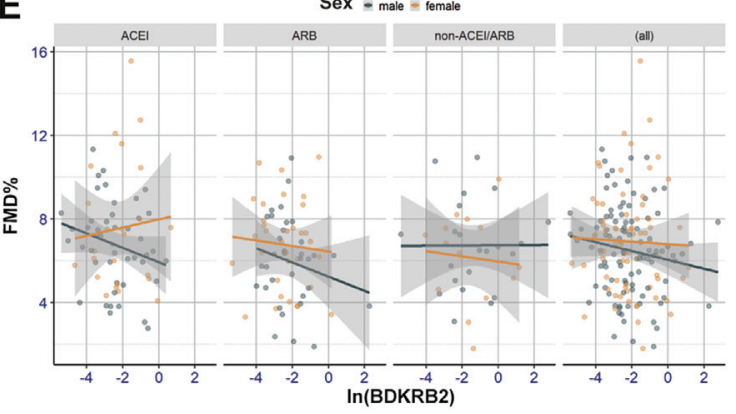

Sex $=$ male $=$ female

Figure 3. Correlations analyses between FMD, plasma total nitrite/ nitrate levels and gene expression of BDKRB1/BDKRB2.

was slightly lower than in the ACEI group, but significantly higher as compared to the non-ACE/ARB group (1.00-fold $\pm 0.39, P=0.0003$ ) (Figure 4A). Regarding BDKRB2, the mRNA expression level in the ACEI group (0.22-fold \pm 0.04 ) was significantly lower than in the non-ACE/ARB group (1.00-fold $\pm 0.45, P=0.0136$ ). Again, the ARB group (0.32-fold \pm 0.14$)$, follows the same trend as the ACEI group, was slightly higher than ACEI group, but it is significantly lower as compared to the non-ACE/ARB group (1.00-fold $\pm 0.45, P=0.0228$ ) (Figure 4B). Finally, the ACE mRNA level was unchanged between all groups, ACEI group (0.63-fold \pm 0.06 ), ARB group (0.69-fold \pm 0.08 ), and non-ACE/ARB group (1.00-fold \pm 0.30 ) (Figure $4 \mathrm{C}$ ).

In the context of previously described total plasma nitrite/nitrate concentration and flow mediated dilation, we performed a correlation analysis in relation to bradykinin receptor BDKRB1 and BDKRB2 gene expression. The correlation analyses showed that FMD positive correlates with BDKRB1 in female patients $(P=0.040)$, however, plasma nitrite/nitrate concentration does not correlate neither with BDKRB1 nor with BDKRB2 (Figure 3, Table III).

\section{Discussion}

This work demonstrates that ACEI, as compared to ARB treatment and non-ACEI/ARB treatment, is effective in increasing the plasma total nitrite/nitrate concentration, with concomitant BDKRB1 upregulation and BDKRB2 downregulation in circulating PBMCs of patients with heterogeneous ischemic vascular disease. However, although NO metabolism was elevated in the ACEI treatment group, endothelial function as measured by FMD remained unchanged as compared to the ARB and Non-ACEI/ARB group, respectively. Furthermore, this study compared nitrite/nitrate levels and FMD to age and demonstrated that FMD but not nitrite/nitrate negatively correlates with age.

Nitrite/nitrates are viewed as the end metabolites of nitric oxide (NO), which is the primary vasodilator molecule and the secondary messenger of the bradykinin receptor signaling pathway. NO not only plays an important role for modulating endothelium-dependent relaxation, but also for regulating active vascular growth [19]. Previous research indicated the superiority of ACEI over ARB with regard to their effect on NO production [20, 21]. In-vivo studies have shown that the nitric oxide synthesis (NOS) inhibitor - L-NAME (N(gamma)-nitroL-arginine methyl ester) causes an overactivation of the renin-angiotensin system (RAS), thereby leading to hypertension. However, ACE knockout mice are resistant to the hypertension induced by L-NAME [22]. Furthermore, administration of ACEI decreased serum asymmetric dimethylarginine, which is an endogenous NOS inhibitor $[23,24]$. These results demonstrate that inhibition of ACE is an important and targeted molecular therapeutic option to upregulate NO levels. These publications are consistent with our finding regarding ACEI treatment elevated plasma NO levels. However, in this study we show for the first time an elevated plasma NO level in hypertensive, ACEI treated patients with different and heterogeneous ischemic vascular diseases (CAD, PAD and CVD). The elevated 
Table III. Correlation analyses

\begin{tabular}{|c|c|c|c|c|c|c|c|c|}
\hline & \multicolumn{2}{|c|}{ ACEI $(n=73)$} & \multicolumn{2}{|c|}{ ARB $(n=68)$} & \multicolumn{2}{|c|}{ Non-ACEl/ARB $(n=36)$} & \multicolumn{2}{|c|}{ Total sample $(n=177)$} \\
\hline & Male & Female & Male & Female & Male & Female & Male & Female \\
\hline \multicolumn{9}{|c|}{ FMD_nitrite/nitrate } \\
\hline r & -0.125 & -0.051 & -0.190 & 0.267 & -0.087 & 0.358 & -0.096 & 0.157 \\
\hline$P$ & 0.402 & 0.805 & 0.282 & 0.126 & 0.700 & 0.210 & 0.336 & 0.181 \\
\hline \multicolumn{9}{|c|}{ FMD_BDKRB1 } \\
\hline r & 0.109 & 0.280 & -0.143 & 0.078 & 0.416 & 0.128 & 0.046 & 0.246 \\
\hline$P$ & 0.476 & 0.175 & 0.442 & 0.672 & 0.076 & 0.678 & 0.657 & 0.040 \\
\hline \multicolumn{9}{|c|}{ FMD_BDKRB2 } \\
\hline r & -0.235 & 0.085 & -0.198 & -0.079 & 0.004 & -0.086 & -0.149 & -0.032 \\
\hline$P$ & 0.120 & 0.688 & 0.261 & 0.663 & 0.986 & 0.769 & 0.137 & 0.787 \\
\hline \multicolumn{9}{|c|}{ Nitrite/nitrate_BDKRB1 } \\
\hline r & -0.042 & -0.061 & 0.138 & 0.217 & -0.304 & 0.001 & 0.037 & 0.097 \\
\hline$P$ & 0.782 & 0.774 & 0.461 & 0.233 & 0.206 & 0.996 & 0.724 & 0.425 \\
\hline \multicolumn{9}{|c|}{ Nitrite/nitrate_BDKRB2 } \\
\hline r & -0.042 & -0.061 & 0.138 & 0.217 & -0.304 & 0.001 & -0.193 & 0.034 \\
\hline$P$ & 0.782 & 0.774 & 0.461 & 0.233 & 0.206 & 0.996 & 0.053 & 0.775 \\
\hline
\end{tabular}

Table IV. Parameters of treadmill test

\begin{tabular}{|c|c|c|c|c|c|c|c|}
\hline & $\begin{array}{l}\text { Maximal walking } \\
\text { distance }(\mathrm{m})\end{array}$ & $\begin{array}{l}\text { Duration } \\
\text { (mm:ss) }\end{array}$ & $\begin{array}{c}\text { Walking } \\
\text { speed }(\mathrm{m} / \mathrm{h})\end{array}$ & $\begin{array}{l}\text { Peripheral } \\
\text { fatigue }\end{array}$ & $\begin{array}{l}\text { Angina } \\
\text { pectoris }\end{array}$ & Dyspnea & Others \\
\hline ACEI $(n=34)$ & $257.97 \pm 192.16$ & $05: 13 \pm 03.55$ & $3036.21 \pm 427.65$ & 8 & 3 & 28 & 6 \\
\hline ARB $(n=38)$ & $250.37 \pm 175.52$ & $05: 06 \pm 03: 31$ & $2966.95 \pm 581.42$ & 14 & 1 & 28 & 5 \\
\hline Non-ACEI/ARB $(n=11)$ & $298.73 \pm 177.90$ & $05: 44 \pm 03: 28$ & $3174.09 \pm 162.97$ & 4 & 0 & 5 & 2 \\
\hline$P$ & 0.741 & 0.880 & 0.453 & 0.443 & 0.343 & 0.055 & 0.846 \\
\hline
\end{tabular}

nitrite/nitrate levels found in this study have interesting scientific implications, $\mathrm{NO}$ is the most important molecule for the regulation of vascular tone, but also plays a crucial role (I) in modulating endothelial function, and (II) in regulating arteriogenesis $[1,5]$.

With regard to endothelial function, recent studies have suggested that endothelial dysfunction precedes the progression of atherosclerosis and other age-related vascular diseases. Therefore, endothelium-dependent FMD can be regarded as a prognostic indicator of major adverse cardiovascular and cerebrovascular events (MACCE) [25]. Indeed, studies measuring FMD in patients with cardiovascular disease showed that endothelial dysfunction correlates to CAD severity [26]. Furthermore, both pre-clinical studies and clinical trials have clearly illustrated that ACEI could improve FMD and prevent endothelial dysfunction $[27,28]$. However, an ACEI related FMD improvement was not confirmed in this analysis. FMD is slightly increased in the ACEI treatment group as compared to the ARB treatment group, but this value is not significantly different. The reason for our deviating findings probably depends on the selection of patients with different underlying cardiovascular diseases. Endothelial function precisely maps biological age, but is also very sensitive to different forms of cardiovascular diseases. Therefore, selection of a heterogeneous group of patients with different cardiovascular diseases could affect endothelial function differently. Nevertheless, there was a clear negative correlation of FMD with patient age. Indeed, numerous studies have shown that progressive endothelial dysfunction is one of the most important aging-associated determinants $[29,30]$, and we confirmed that FMD is strongly and negatively correlated with increasing age. Since, we enrolled the patients in a randomized manner, the number of males and females differed by $58.19 \%$ to $41.81 \%$. We therefore also investigated the differences in nitrite/nitrate levels and FMD between males and females. In our analysis there were no differences in nitrite/nitrate levels and FMD related to gender, thus different gender ratios within our patient group should not bias the group differences discussed herein.

In addition, our results show for the first time that patients on ACEI medication have upregulated BDKRB1, but downregulates BDKRB2 mRNA expression in PBMCs. ACEI not only inhibit angiotensin I (Ang I) to angiotensin II (Ang II) conversion, but also increase bradykinin concentration. Bradykinin derivatives are direct ligands of BDKRB2 and BDKRB1. In fact, stimulation of BDKRB2 leads to intracellular activation of endothelial nitric oxide synthase (eNOS) in the endothelia cell layer, and stimulation of BDKRB1 results in activation of inducible nitric oxide synthase (iNOS) in immune cells, such as PBMCs 

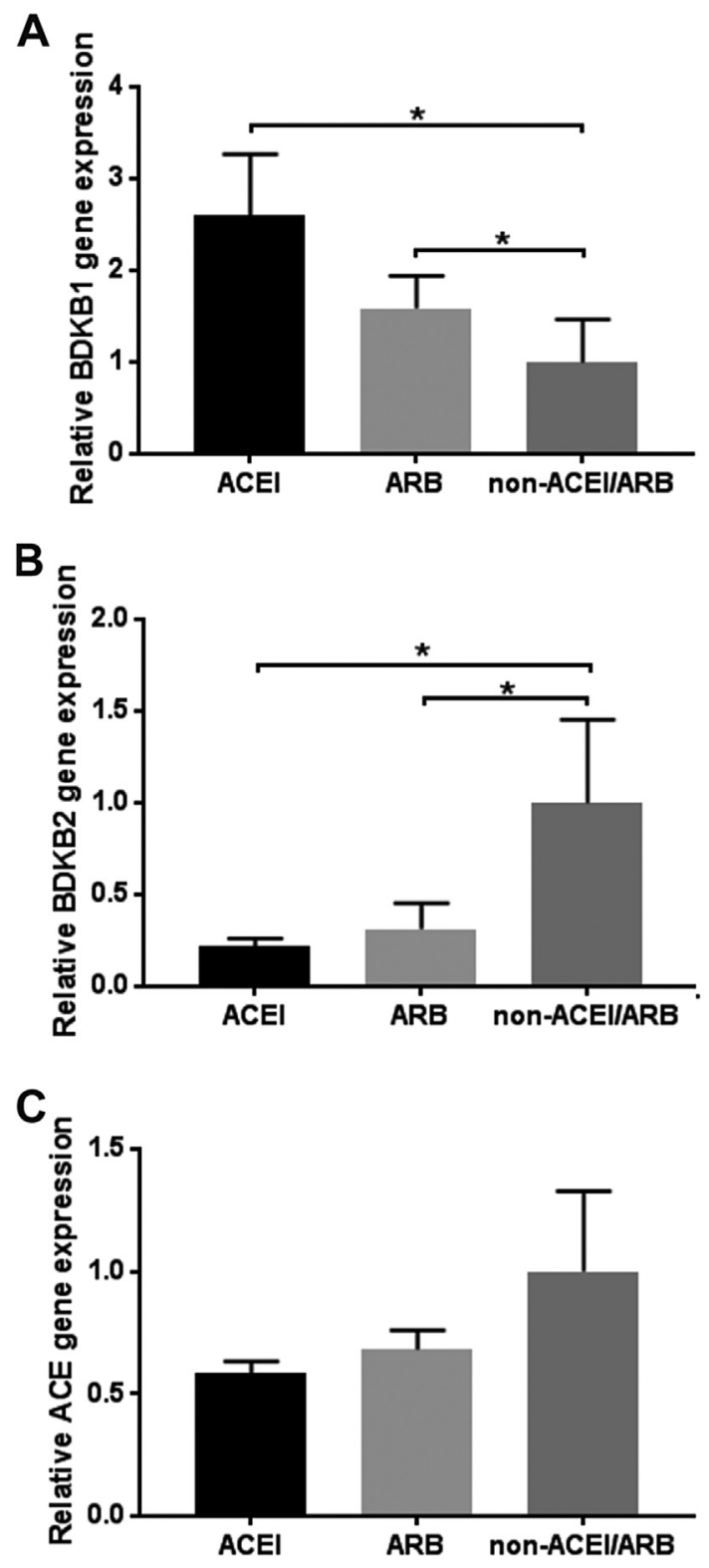

Figure 4. Gene expression of BDKRB1: ACEI $(n=70)$, ARB $(n=63)$, nonACEl/ARB ( $n=32)$, gene expression of BDKRB2: ACEI $(n=70), A R B$ $(n=67)$, non-ACEI/ARB $(n=36)$, and gene expression of ACE: ACEI $(n=72), \operatorname{ARB}(n=68)$, non-ACEI/ARB $(n=36)$ in PBMC $\left({ }^{*} P \leq 0.05\right)$.

[14]. Previous research has also shown that the antagonist of BDKRB2 attenuated the augmentation of NO production induced by ACEI. While BDKRB2s are known to regulate vascular tone, they also exhibit atherogenic activity by increased reactive oxygen species generation [31]. In contrast, BDKRB1s exhibit anti-atherosclerotic activities, and mice deficient in kinin B1 receptor and apolipoprotein E showed a predisposition to atherosclerosis development [32]. Recent research shows that both of BDKRB1 and BDKRB2 can respectively bind to different heterotrimeric $G$ proteins and thereby increase NO production by activing either iNOS or eNOS: in normal physiological conditions, BDKRB2 couples through $G_{\alpha q} / 11$ and results in calmodulin-dependent activation of eNOS; whereas, in the inflammatory conditions (myocardial infarction, ischemic stroke, acute peripheral arterial occlusion), B1R couples through $G_{\alpha \mathrm{i}}, \mathrm{G}_{\beta \gamma}$ and results in activation of iNOS through the ras-raf-mek-erk-map kinase pathway [14]. Hence, the putative ACEI-BDKRB1-iNOS and ACEIBDKRB2-eNOS signaling pathways may be of significance as a target for anti-atherogenic and pro-arteriogenic therapies in future.

Indeed, accumulating evidence suggests that BDKRB1 are amplified and expressed constitutively in PBMC subtypes such as macrophages [33]. Our research group demonstrated, that in particular BDKRB1 signaling regulates arteriogenesis by modulation of monocyte/macrophage migration. Moreover, we tested whether bradykinin receptors play a functional role in adaptive arteriogenesis, and analyzed the role of the bradykinin receptor system for collateral artery growth by using a loss-of-function approach. We found a strong reduction in peripheral arteriogenesis for $\mathrm{BDKRB}^{-/-}$mice and only a minor reduction for $\mathrm{BDKRB} 2^{-1-}$ mice. A transplantation of wild type into $\mathrm{BDKRB}^{-/-}$mice recovered the loss of arterial flow after an arterial occlusion surgery, as seen in untreated BDKRB1 mutant mice (gain of function). The enhanced expression of BDKRB1 on immune cells seems to be the pivotal determinant during collateral artery growth and vascular regeneration [4]. Our finding that ACEI treatment might activate BDKRB1 has a clinically perspective and evidence is growing that BDKRB1 can be activated by ACEI directly on the extracellular loop without altering conformational change [12]. Interestingly, our correlation analyses between bradykinin receptor expression and endothelial function shows that there is a positive correlation between FMD and BDKRB1 exclusively in female patients. This is indeed consistent with the findings of $\mathrm{Wu}$ et al. [34], who previously speculated here that sex differences in BDKRB1induced cytokine production may occur in response to ACEI therapy. Indeed, our results here also suggest a possible sex-specific difference in BDKRB1-mediated endothelial function in response to ACEI therapy that needs to be further investigated.

\section{Limitations}

The current cross-sectional study is aimed at analyzing the gene expression of bradykinin receptors and ACE in peripheral blood mononuclear cells from patients with ACEI or ARB treatment. Here, we did not conduct any new pharmacological therapy, therefore, the evidence level grade is lower than that of a randomized clinical trial (RCT) or cohort study.

\section{Conclusions}

We firstly showed that ACEI treatment enhances total nitrite/nitrate concentration in patients with underlying heterogenous cardiovascular diseases. NO production might also enhance endothelial function in the ACEI treatment group as previously reported, however FMD 
measurements showed no differences between the medication treatment groups. The selection of patients with different and concomitant cardiovascular diseases and risk status might influence FMD analysis. FMD is known to be strongly affected by different risk factor such as hypertension, diabetes or severity of atherosclerosis status [25]. Indeed, we demonstrated that FMD is strongly negatively correlated with age in patients with concomitant ischemic vascular disease (CAD, PAD and CVD) and hypertension. Hence, FMD is sensitive to underlying heterogenous cardiovascular diseases, and was again shown to be a strong determinant of vascular aging.

Secondly, ACEI treatment induces the upregulation of BDKRB1, but the downregulation of BDKRB2 mRNA expression in PBMC from patients with cardiovascular disease. The role ACEI-bradykinin receptor signaling pathway is expected to redirect away from pathologic atherosclerosis towards physiological arteriogenesis, which is worthy of further investigation.

\section{References}

1. Zietzer A, Buschmann EE, Janke D, Li L, Brix M, Meyborg $H$, et al. Acute physical exercise and long-term individual shear rate therapy increase telomerase activity in human peripheral blood mononuclear cells. Acta Physiol. 2017;220(2):251-62.

2. Buschmann I, Schaper W. Arteriogenesis versus angiogenesis: two mechanisms of vessel growth. News Physiol Sci. 1999;14: $121-5$.

3. Buschmann IR, Busch HJ, Mies G, Hossmann KA. Therapeutic induction of arteriogenesis in hypoperfused rat brain via granulocyte-macrophage colony-stimulating factor. Circulation. 2003;108(5):610-5.

4. Hillmeister P, Gatzke N, Dulsner A, Bader M, Schadock I, Hoefer I, et al. Arteriogenesis is modulated by bradykinin receptor signaling. Circ Res. 2011;109(5):524-33.

5. Brix M, Buschmann EE, Zietzer A, Jaurigue JA, Li L, Jungk C, et al. Long-term individual shear rate therapy counterpulsation enhances plasma nitrite release in patients with PAD. Vasa. 2017;46(1):37-45.

6. Knuuti J, Wijns W, Saraste A, Capodanno D, Barbato E, FunckBrentano C, et al. 2019 ESC Guidelines for the diagnosis and management of chronic coronary syndromes. Eur Heart J. 2020;41(3):407-77.

7. Aboyans V, Ricco JB, Bartelink MEL, Björck M, Brodmann M, Cohnert T, et al. 2017 ESC Guidelines on the Diagnosis and Treatment of Peripheral Arterial Diseases, in collaboration with the European Society for Vascular Surgery (ESVS): Document covering atherosclerotic disease of extracranial carotid and vertebral, mesenteric, renal, upper and lower extremity arteries Endorsed by: the European Stroke Organization (ESO) The Task Force for the Diagnosis and Treatment of Peripheral Arterial Diseases of the European Society of Cardiology (ESC) and of the European Society for Vascular Surgery (ESVS). Eur Heart J. 2018;39(9):763-816.

8. Ponikowski P, Voors AA, Anker SD, Bueno H, Cleland JGF, Coats AJS, et al. 2016 ESC Guidelines for the diagnosis and treatment of acute and chronic heart failure: The Task Force for the diagnosis and treatment of acute and chronic heart failure of the European Society of Cardiology (ESC) Developed with the special contribution of the Heart Failure Association (HFA) of the ESC. Eur Heart J. 2016;37(27):2129-200.

9. Williams B, Mancia G, Spiering W, Agabiti Rosei E, Azizi M, et al. 2018 ESC/ESH Guidelines for the management of arterial hypertension. Eur Heart J. 2018;39(33):3021-104.
10. Manolis AJ, Marketou ME, Gavras I, Gavras H. Cardioprotective properties of bradykinin: role of the $\mathrm{B}(2)$ receptor. Hypertens Res. 2010;33(8):772-7.

11. Auch-Schwelk W, Bossaller C, Claus M, Graf K, Gräfe M, Fleck E. ACE inhibitors are endothelium dependent vasodilators of coronary arteries during submaximal stimulation with bradykinin. Cardiovasc Res. 1993;27(2):312-7.

12. Erdos EG, Tan F, Skidgel RA. Angiotensin I-converting enzyme inhibitors are allosteric enhancers of kinin B1 and B2 receptor function. Hypertension. 2010;55(2):214-20.

13. Hillmeister $P$, Bondke Persson A. Bradykinin-from snake poison to therapeutic options. Acta Physiol. 2020;228(3):e13445.

14. Kuhr F, Lowry J, Zhang Y, Brovkovych V, Skidgel RA. Differential regulation of inducible and endothelial nitric oxide synthase by kinin B1 and B2 receptors. Neuropeptides. 2010;44(2):145-54.

15. Burnier M. Angiotensin II type 1 receptor blockers. Circulation. 2001;103(6):904-12

16. Carey RM, Wang ZQ, Siragy HM. Role of the angiotensin type 2 receptor in the regulation of blood pressure and renal function. Hypertension. 2000;35(1 Pt 2):155-63.

17. Abadir PM, Periasamy A, Carey RM, Siragy HM. Angiotensin II type 2 receptor-bradykinin B2 receptor functional heterodimerization. Hypertension. 2006;48(2):316-22.

18. Shen B, Harrison-Bernard LM, Fuller AJ, Vanderpool V, Saifudeen Z, El-Dahr SS. The Bradykinin B2 receptor gene is a target of angiotensin II type 1 receptor signaling. J Am Soc Nephrol. 2007;18(4):1140-9.

19. Prior BM, Lloyd PG, Ren J, Li Z, Yang HT, Laughlin MH, et al. Arteriogenesis: role of nitric oxide. Endothelium. 2003;10(4-5): 207-16.

20. Imanishi T, Kuroi A, Ikejima H, Kobayashi K, Muragaki Y, Mochizuki S, et al. Effects of angiotensin converting enzyme inhibitor and angiotensin II receptor antagonist combination on nitric oxide bioavailability and atherosclerotic change in Watanabe heritable hyperlipidemic rabbits. Hypertens Res. 2008;31(3):575-84.

21. Wilmink HW, Banga JD, Hijmering $M$, Erkelens WD, Stroes ES, Rabelink TJ. Effect of angiotensin-converting enzyme inhibition and angiotensin II type 1 receptor antagonism on postprandial endothelial function. J Am Coll Cardiol. 1999;34(1):140-5.

22. Giani JF, Janjulia T, Kamat N, Seth DM, Blackwell WL, Shah $\mathrm{KH}$, et al. Renal angiotensin-converting enzyme is essential for the hypertension induced by nitric oxide synthesis inhibition. J Am Soc Nephrol. 2014;25(12):2752-63.

23. Ito A, Egashira $K$, Narishige $T$, Muramatsu $K$, Takeshita A. Angiotensin-converting enzyme activity is involved in the mechanism of increased endogenous nitric oxide synthase inhibitor in patients with type 2 diabetes mellitus. Circ J. 2002;66(9):811-5.

24. Chen JW, Hsu NW, Wu TC, Lin SJ, Chang MS. Long-term angiotensin-converting enzyme inhibition reduces plasma asymmetric dimethylarginine and improves endothelial nitric oxide bioavailability and coronary microvascular function in patients with syndrome X. Am J Cardiol. 2002;90(9): 974-82.

25. Deanfield JE, Halcox JP, Rabelink TJ. Endothelial function and dysfunction: testing and clinical relevance. Circulation. 2007;115(10):1285-95.

26. Sancheti S, Shah P, Phalgune DS. Correlation of endothelial dysfunction measured by flow-mediated vasodilatation to severity of coronary artery disease. Indian Heart J. 2018;70(5): 622-6.

27. Varin R, Mulder P, Tamion F, Richard V, Henry JP, Lallemand $F$, et al. Improvement of endothelial function by chronic angiotensin-converting enzyme inhibition in heart failure: role of nitric oxide, prostanoids, oxidant stress, and bradykinin. Circulation. 2000;102(3):351-6.

28. Ceconi C, Fox KM, Remme WJ, Simoons ML, Bertrand M, Parrinello G, et al. ACE inhibition with perindopril and endothelial function. Results of a substudy of the EUROPA study: PERTINENT. Cardiovasc Res. 2007;73(1):237-46. 
29. Maruhashi T, Soga J, Fujimura N, Idei N, Mikami S, Iwamoto Y, et al. Relationship between flow-mediated vasodilation and cardiovascular risk factors in a large community-based study. Heart. 2013;99(24):1837-42.

30. Yeboah J, Crouse JR, Hsu FC, Burke GL, Herrington DM. Brachial flow-mediated dilation predicts incident cardiovascular events in older adults: the Cardiovascular Health Study. Circulation. 2007;115(18):2390-7.

31. Perhal A, Wolf S, Jamous YF, Langer A, Abd Alla J, Quitterer U. Increased reactive oxygen species generation contributes to the atherogenic activity of the B2 bradykinin receptor. Front Med. 2019;6:32.

32. Merino VF, Todiras M, Mori MA, Sales VM, Fonseca RG, Saul V, et al. Predisposition to atherosclerosis and aortic aneurysms in mice deficient in kinin B1 receptor and apolipoprotein E. J Mol Med. 2009;87(10):953-63.

33. Bockmann S, Paegelow I. Kinins and kinin receptors: importance for the activation of leukocytes. J Leukoc Biol. 2000; 68(5):587-92.

34. Wu H, Roks AJ, Leijten FP, Garrelds IM, Musterd-Bhaggoe UM, van den Bogaerdt AJ, et al. Genetic variation and gender determine bradykinin type 1 receptor responses in human tissue: implications for the ACE-inhibitor-induced effects in patients with coronary artery disease. Clin Sci. 2014;126(6): 441-9.

\section{History}

Submitted: 15.05.2021

Accepted after revision: 31.07.2021

Published online: 25.08.2021

\section{Acknowledgement}

This study was supported by the Ministry of Science, Research and Culture, Federal State of Brandenburg (Ministeriums für Wissenschaft, Forschung und Kultur des Landes Brandenburg), the Federal Ministry of Education and Research (Bundesministerium für Bildung und Forschung) and the Chinese Scholarship Council (CSC).

\section{Conflict of interest}

The authors have declared no conflict of interest.

\section{Correspondence address}

\section{Philipp Hillmeister, PhD}

Brandenburg Medical School (MHB)

Faculty of Health Sciences (FGW)

Center for Internal Medicine I.

Department for Angiology \& WalkByLab

Campus University Clinic Brandenburg

Hochstrasse 29

14770 Brandenburg an der Havel

Germany

p.hillmeister@klinikum-brandenburg.de 